\title{
Knowledge of menstrual hygiene management among adolescent girls: what does evidence show?
}

\author{
Niki Shrestha1, Ganesh Dangal2, Gayatri Khanal, Tulsi Ram Bhandari4 \\ 1Department of Community Medicine, Chitwan Medical College, Nepal \\ ${ }_{2}$ Department of Obstetrics and Gynecology, Kathmandu Model Hospital, Kathmandu, Nepal \\ ${ }_{3}$ School of Public Health, Chitwan Medical College, Nepal \\ ${ }_{4}$ Department of Public Health, School of Health and Allied Sciences, Pokhara University, Nepal
}

Received: May 19, 2020

Accepted: May 31, 2020

\section{ABSTRACT}

\begin{abstract}
Aims: To explore the knowledge of menstrual hygiene management among adolescent girls in different domains like culture, social learning and belief.

Methods: This review paper explored the knowledge of menstrual hygiene management under several domains. Literature published in Pub Med, Medline, Google Scholar and HINARI from 2010 to 2020 was reviewed to prepare this paper.

Results: The studies revealed that there is a general silence about menstruation. Adequate and accurate information about menstruation and the need for good menstrual hygiene management is limited. It further revealed that girls are not provided with comprehen sive information before menarche while growing up, and therefore the first experience of menstruation is without any preparation and usually comes as a shock. Menstruation is widely regarded as a curse from God or as a disease or as being impure or as a taboo. Schools are doing very little to impart education on Menstrual Hygiene Management to adolescents. A majority of the teachers themselves are least or unprepared to address this issue with the girls, even when it is part of the school curriculum. The studies provide a wealth of information for designing interventions to improve menstrual hygiene management.
\end{abstract}

Conclusions: Young girls are not well aware of the menstrual event and the hygienic measures. They are neither well informed nor properly taught by elders and teachers. Effective interventions must be contextualized, designed, and implemented to generate awareness and change the mindset of people surrounding menstruation.

Keywords: adolescents, knowledge, menstrual hygiene management, reproductive health

Citation : Shrestha N, Dangal G, Khanal G, Bhandari TR. Knowledge of menstrual hygiene management among adolescent girls: what does evidence show? Nep J Obstet Gynecol. 2020;15(30):9-17. DOI: 10.3126/njog.v15i1.29333

\section{INTRODUCTION}

Menstruation is a natural physiological phenomenon in healthy adolescent girls and pre-menopausal women.1 The hygienic management of menstruation is vital for girls and women to participate in society with comfort and dignity. Adequate menstrual hygiene management (MHM) has been defined as - using hygienic menstrual management material to absorb menstrual blood; that can be changed in privacy as and when required, for the duration of a menstrual period; using soap and water for washing as required; having access to convenient as well as safe facilities for disposing of used menstrual management materials; comprehending the basic facts associated to the menstrual cycle and managing it with dignity and without fear or discomfort.2

The provision of adequate knowledge on menstruation before menarche could make young females view menstruation as an important milestone in their lives and just a natural phenomenon. Safe and effective menstrual health management is a vital component of, and premise to, adolescent girls' sexual and reproductive health. If women and girls have knowledge about their body and the basic facts about fertility, and if they are thereby able to efficiently manage their menstrual hygiene, they may

\section{CORRESPONDENCE}

Dr Niki Shrestha

Department of Community Medicine, Chitwan Medical College,

Nepal Email: shrestha.niki@cmc.edu.np; Phone: +977-9851202040 
consequently be empowered and more equipped with the knowledge, the skill and the confidence which is necessary to manage their long term sexual and reproductive health. 3 Poor knowledge on menstruation subsequently renders women and girls unable to manage menstrual periods efficiently and this, in turn, renders them vulnerable to the risk of contracting reproductive tract infections, urinary tract infections and pelvic inflammatory diseases.4 Imparting a girl child with the knowledge about menstruation builds up better attitude towards menstruation.5

Studies have shown that girls with good knowledge of menstruation faired better in practicing good menstrual hygiene management.6 Adequate and accurate menstrual knowledge is paramount to ensuring positive health outcomes. Some studies suggest that girls are often unaware about menstruation before menarche which can consequently lead to anxiety, embarrassment and lack of hygienic menstrual management practices.79 Besides, understanding the beliefs, attitudes, and perceptions about menstruation, often influenced by cultural and social norms are paramount to enhancing correct menstrual practices and safeguarding the health of women and girls.7 Menarche at an earlier age and a special focus on education means that several adolescent girls are in school while menstruating. Girls will experience menstrual bleeding on at least some school days every month when there is a typical menstrual cycle lasting 25 to 30 days within which bleeding occurs for 4 to 6 days. Therefore, we can say that menstrual hygiene management is an increasingly important though often unrecognized issue which is heavily interlaced with girls' education, empowerment, and overall social development.10

A recent systematic review of women's and girls' experiences of menstruation in low- and middleincomecountries highlighted that interventions for MHM must address a varied range of issues such as knowledge, restrictive behavioural expectations, addressing stigma, social support, and the physical and economic environment.11 Therefore, in alignment with this, the term menstrual health and hygiene (MHH) encompasses both MHM and the broader systemic factors which link menstruation with overall wellbeing, equity, gender equality, education, empowerment, and rights.2 The objective of this review paper is to assess the following outcomes among adolescent girls - awareness of menarche before the start of menstruation; frequently cited source of information about menstruation; knowledge of menstruation as a physiological process/biological event; knowledge of MHM materials/absorbents; misconceptions about menstruation e.g. menstruation is a disease/curse from God, restrictions should be followed during menstruation; menstruation is impure, etc.; anticipation of menarche e.g. embarrassment, shame, fear, anxiety, and so on.

\section{METHODS}

For preparing this review paper on Knowledge of Menstrual Hygiene Management (MHM), we conducted a detailed online search in the first and second weeks of May 2020. We searched observational studies with information on MHM, considering the knowledge, attitude, perceptions among adolescent (10-19 years) girls in Nepal, South-East Asia, African Countries, Middle-East, low and middle income (LMIC) countries published between 2010 and April 2020. We used PubMed, Google Scholar, Medline and HINARI webpages using the keywords "adolescent", "menstrual", "menstrual hygiene management", "Knowledge on menstrual hygiene management", "South-East Asia", "Middle East" and "African Countries". We searched for the 'grey literature': technical reports, documents, published study reports and blogs. Other relevant studies were also identified by retrieving references of included studies. The last search was conducted on May 14, 2020. We selected 100 articles and 20 grey literature. Out of 100 articles, we excluded 44 articles for not exactly meeting the objectives of the study and finally selected 56 articles for this review study [Figure-1]. 


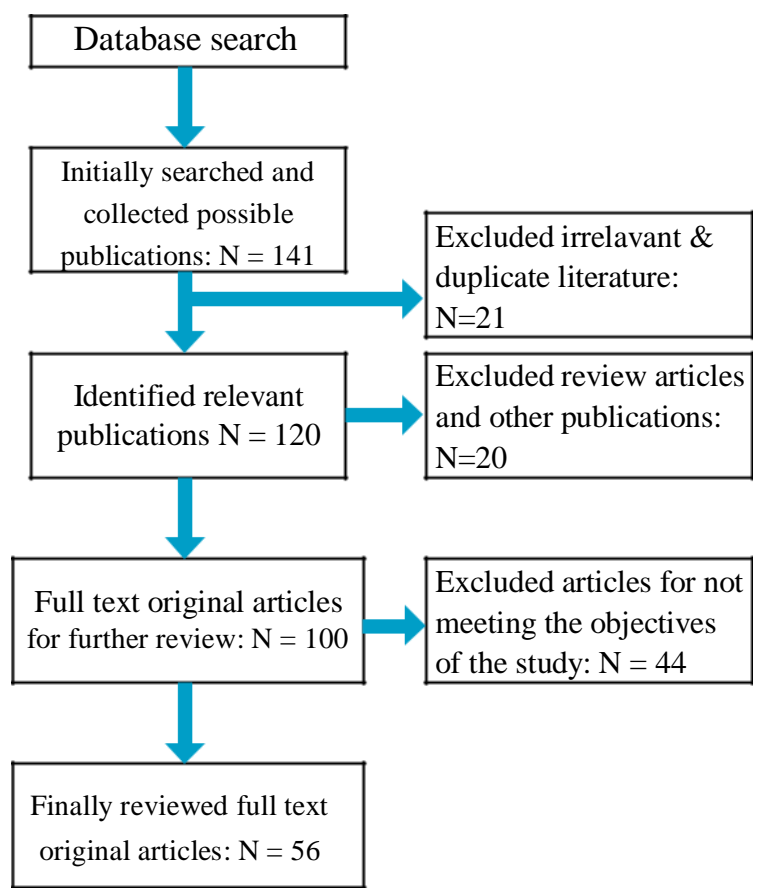

Figure-1: Prisma flow diagram for the selection of relevant articles

We reviewed only the full texts, quantitative, qualitative andmixed method studies which were conducted in South-East Asia, Middle East, African countries, and other LMICs. Only those studies are included which were accessible electronically until March 2020. The only peer reviewed articles written in English are included in this review. 'Grey literature' such as technical reports, documents, published study reports, blogs, were included. Study participants included were adolescent females aged 10-19 years. Only those articles published between 2010 to March 2020 were included.

We measured at least one of these outcomes: awareness of menarche before the start of menstruation; frequently cited source of information about menstruation; knowledge of menstruation as a physiological process/biological event; knowledge of MHM materials/absorbents; misconceptions about menstruation e.g. menstruation is a disease/ curse from God, restrictions should be followed during menstruation; menstruation is impure, etc.; anticipation of menarche e.g. embarrassment, shame, fear, anxiety, and so on. Editorials, theoretical papers, case study, review papers, essays, letters, opinion pieces, and newspaper articles were excluded.

\section{RESULTS}

\section{Knowledge of menstruation as a physiological process}

In Nepal, prior studies reported the knowledge of menstruation as a physiological process from the lowest response rate of 36\% (Sapkota et al) 12 to the highest response rate of $94.8 \%$ (Neupane et al).13 Among South-East Asian Countries, only 19\% of study participants in Pakistan in a study conducted by Ali et a $1_{14}$ knew about menstruation being a physiological process. Studies reported the lowest response rate (10\%) in a study conducted by Santra et al 15 in a slum of Kolkata, West Bengal, India. Upto $89.7 \%$ of participants had knowledge on menstruation as a physiological process in another study in India by Shoor et al ${ }_{16}$ conducted in an urban field practice area of a medical college in Uttar Pradesh, India. A study conducted in Bangladesh by Anee et al ${ }_{17}$ among

girls in Madrasa reported that only 33.33\% of girls knew about menstruation as a physiological process. Among the African countries, response rates ranged from $57.89 \%$ in a study conducted in Northeast Ethiopia by Tegegne et al ${ }_{18}$ to a highest of $99.2 \%$ in South-Western Nigeria in a study conducted by Aluko et al.19

\section{Knowledge of menstruation before menarche}

A study by Morrison et al 20 conducted in five districts of Nepal revealed that only $24 \%$ of study participants knew about menstruation before attaining menarche. In another study by Rajbhandari et $\mathrm{al}_{21}$, the response rate was $64.90 \%$. In African countries, the response rate ranged from $51.5 \%$ in a study conducted in the rural Gambia by Shah et al 22 to $86.75 \%$ in a study conducted in Northeast Ethiopia by Tegegne et al.18 In southeast Asia, the response rate was alarmingly as low as $15 \%$ in a study in Tamil Nadu, India conducted by Varghese et al.23In middle east countries, the response rate ranged from $41 \%$ in Yemen, in a study conducted by Mohamed et $\mathrm{al}_{24}$ to $62.5 \%$ in a study conducted in Saudi Arabia by Bano et al.25

\section{Knowledge of MHM practices and infection prevention}

The commercial sanitary pad was known to $49.18 \%$ of the respondents in Ethiopia in a study conducted by Gultie et al.26 However, in another study in Nigeria by Aluko et al,19 upto $98 \%$ of the study participants 
responded that sanitary pad is the best sanitary material to be used for menstruation. In a study conducted in Ghana by Yiadom et $\mathrm{al}_{27}, 80.7 \%$ of the study participants were aware of sanitary pads, $22 \%$ were aware of tampons and $7.4 \%$ were aware of menstrual cups. In South-East Asia, only 39\% of the study participants were aware of the use of sanitary pads in Haryana, India in a study conducted by Kamboj et al.28 In Nepal, the response rate ranged from $67.2 \%$ in rural Nepal in a study conducted by Sapkota et al ${ }_{12}$ to $90 \%$ in a study conducted by Yadav et al.29 A study in Nepal conducted by Dahal et al 30 in Jumla district, revealed that $75.9 \%$ of the adolescent school girls reported having knowledge that poor MHM predisposes to infections. In another study conducted in Saudi Arabia by Bano et $\mathrm{al}_{25}$, the response rate was $80 \%$. The response rate was $86.5 \%$ in a study conducted in India by Balat et al 31 among adolescent school girls in Ahmedabad City. A study in Tamale, Ghana by Ameade et al 32 among university students reported a response rate as high as $95.9 \%$.

\section{Misconceptions regarding menstruation}

There were 13 studies about a misconception that menstruation is a curse from God. The response rate was as low as $1 \%$ in a study in Nepal conducted by Yadav et $\mathrm{al}_{29}$ and in a study in Bangladesh conducted by Alam et al $_{33}$ to as high as $28 \%$ reported in a study in Western Ethiopia conducted by Upashe et $\mathrm{al}_{34}$ and in another study conducted in India by Raina et al.35 In several studies, the study participants were asked if menstrual blood is impure or if menstruating girl is unclean. In Nepal, the response rate for this domain was as low as $11.5 \%$ in a study conducted by Sapkota et $\mathrm{al}_{12}$ to as high as $82.4 \%$ in a study conducted by Shrestha et al.36 In southeast Asia, the response rate was $19 \%$ in Pakistan in a study conducted by Ali et ali4in urban Karachi. In Indonesia, the response rate was $34 \%$ in a study conducted by Davis et al.37 In a study in rural Kheda district of Gujarat, India, conducted by Prajapati et $\mathrm{al}_{38}$ the response rate for this domain was as high as $76 \%$. Another study from Ghana by Yiadom et $\mathrm{al}_{27}$ revealed that $70 \%$ of the study participants reported that a girl is unclean during menstruation. A study from Yemen by Mohamed et $\mathrm{al}_{24}$ revealed that $37.8 \%$ of the study participants reported the same.
Several studies reported the study participants having the misconception that menstruation is a disease. In South-East Asia, study participants in urban Karachi in Pakistan in a study by Ali et al ${ }_{14}$ reported a response rate of as low as $1.8 \%$. The response rate was $6.5 \%$ in Nepal in a study conducted among adolescent school girls of Bhaktapur, Nepal by Rajbhandari et al.21 A study conducted by Panditet al 39 among adolescent school girls in rural areas of West Bengal, India revealed a response rate of $14.4 \%$. A high response rate of $35.2 \%$ was reported in the rural Gambia by Shah et al.22 Although there are several studies about the practices of various types of cultural restrictions during menstruation, only a few quantitative studies have studied the attitude and belief regarding whether there should be any restrictions during menstruation. In a study in 5 districts of Nepal by Morrison et al20, a total of $51.2 \%$ of the study participants responded that they should not visit a religious place due to fear of divine retribution. A total of $14.5 \%$ of study participants also believed that they should not sleep in the same household as others because of fear of divine retribution. In another study in Uttar Pradesh India, conducted by Shoor et al16, $28.76 \%$ of the study participants believed that cultural restrictions should be followed during menstruation.

\section{Anticipation about menarche}

Fear and anxiety at menarche werereported by several studies. In a study in rural Nepal by Sapkota et $\mathrm{al}_{12}$, the reported rate of fear at menarche was $36 \%$. In South-East Asia, the reported rates of fear at menarche ranged from $20 \%$ in a study conducted in Andhra Pradesh, India by Rani et al ${ }_{40}$ to as high as $62.6 \%$ in Pakistan in a study conducted in urban Karachi by Ali et al.14 In African countries, the reported rate ranged from $31.65 \%$ in Ethiopia in a study conducted by Tegegne et al 18 to $65 \%$ in Gambia in a study conducted by Shah et al.22 Embarrassment and shame at the menstruation period werereported by several studies. A study by Rajbhandari et $\mathrm{al}_{21}$ in Nepal reported a response rate of $37.5 \%$. In South-East Asia, the response rate ranged from $19 \%$ in Punjab, India in a study conducted by Kaur et $\mathrm{al}_{41}$ to $30 \%$ in Indonesia in a study conducted by Davis et al.37

\section{Sources of information on menstruation}

Mothers and sisters were cited as the source 
of information and advice for girls regarding menstruation in several studies. The response rate for this knowledge domain was $54 \%$ in a study in the Jumla District of Nepal, conducted by Dahal et al.30 However, in another study conducted by Neupane et al. in Chitwan, Nepal, the response rate for the same, was 81.90\%.13 In African countries, the response rate ranged from $35 \%$ in a study conducted in Ethiopia by Upashe et $\mathrm{al}_{34}$ to $90 \%$ in a study conducted in Ghana by Yiadom et al.27 In the Middle - East, the response rate ranged from $56 \%$ in a study conducted in Saudi Arabia by Bano et al 25 to $75 \%$ in a study conducted in Iran by Poureslami et al.4 In India, the response rate ranged from as low as $12 \%$ in a study conducted by Dhingra et $\mathrm{al}_{42}$ among tribal girls in Jammu and Kashmir, India to as high as $94 \%$ in a study conducted in Nagpur, India by Thakre et al.43Friends were cited as the source of information in several studies. The reported response rate in Nepal ranged from $18 \%$ in a study conducted by Sapkota et $\mathrm{al}_{12}$ to $39 \%$ in a study conducted by Morrison et al.20 In African countries, the response rate ranged from $14 \%$ in a study in Nigeria conducted by Rumun et al 44 to $68 \%$ in a study conducted in Ethiopia by Upashe et al.34 In the Middle East, Bano et al 25 reported a response rate of $17 \%$ in Saudi Arabia and Mohamed et $\mathrm{al}_{24}$ reported a response rate of $25 \%$ in Yemen. In southeast Asia, the response rate ranged from $15 \%$ in Pakistan in a study conducted by Ali et al 14 to as high as $83 \%$ in India in a study conducted by Dhingra et al.42

In southeast Asia, the response rate for teachers and school as the source of information ranged from $7.4 \%$ in a study conducted by Ali et $\mathrm{al}_{14}$ in Pakistan to as high as $47.8 \%$ in a study conducted in India by Thakur et al.45 In African countries, the response rate ranged from $5 \%$ in a study conducted in Ghana by Yiadom et al 27 to as high as $78 \%$ in a study conducted in Gambia by Shah et al.22 In Nepal, the response rate was as low as $8.3 \%$ to $10 \%$ in studies conducted by Neupane et al ${ }_{13}$ and Rajbhandari et $a_{21}$ respectively. Out of all the studies reviewed, only three studies mentioned about health workers being the source of information for the study participants. A study in Nepal conducted by Sapkota et al 12 reported that only $1.6 \%$ of the study participants received information about menstruation from health workers. In another study by Morrison et $\mathrm{al}_{20}, 8 \%$ of the study participants reported receiving information from health works. In India, study participants reported health workers as the source of information with a response rate of $6 \%$ and $7 \%$ in studies conducted by Ramachandra et al ${ }_{46}$ and Thakur et al 45 respectively.

\section{DISCUSSION}

Menstrual Hygiene Management has three inter-linked dimensions - Breaking the silence: adolescent girls should beencouraged to talk and discuss freely menstruation inan open, informed and positive manner to prepare them physically and emotionally for menarche and the menstrual periods; Managing menstruation hygienically:adequate water, cleansing materials and privacy for managing menstrual flows hygienically, and with dignity, both inside homes and in public spaces should be ensured; Safe reuse and disposal solutions: there should be mechanisms for safe reuse, safe collection and disposal of menstrual waste in an environmentally safe manner.47 The study revealed that adequate and accurate information about menstruation and the need for good menstrual hygiene management is limited. Menstruation is not openly and comfortably discussed, particularly with boys and men.47 It further revealed that a majority of girls did not receive the required information about menstruation before menarche.

Qualitative studies revealed that the young girls are mostly not prepared for menstruation before menarche, and therefore menarche is a distressing experience of anxiety, fear, embarrassment, and pain; andgirls are not provided with comprehensive information before menarche while growing up, and therefore the first experience of menstruation is without any preparation and usually comes as a shock.18,22,45,48-50 There is a general silence about menstruation, but one reason for this lack of preparation for menarche seems to be because the parents and adults do not comprehend the need to broach the issue of menstruation to these young girls.48,22 The focus group discussions in these studies have revealed thatelders, teachers and parentsbelieve that menstruation is a normal bodily function and there is nothing remarkable about it, and therefore girls do not need any special explanation or preparatory training for it.This also shows the general lack of understanding of the need for menstrual hygiene management and the role MHM plays for the health and overall wellbeing of girls.48,22 It was evident from these studies that menstruation is generally a secret from men and it appeared that it is 
the sole responsibility of females to impart education as well as information to the girls. It was also evident that these females themselves have misconceptions about menstruation, they also have limited access to the right information and this results in negative ideas and misinformation being passed on tom the younger generation of girls.

It is sad to note from the various studies that teachers and schools are doing very little to impart education on MHM to adolescents. The information given to girls before menarche in the schools is very limited. The qualitative studies reported that a majority of the teachers themselves are least or unprepared to address this issue with the girls, even when it is part of the school curriculum.18,22,45,48-50 The consequent misinformation, misconception and negative attitude and perception towards menstruation can be detrimental in the long run in the sexual and reproductive health journey of a young girl as well as in terms of social development and individual dignity and selfconfidence. Menstruation is a taboo as reported by studies; and there is reluctance in the society to talk openly about sexual and reproductive health, about puberty and menstruation in particular, largely attributable to religious and cultural beliefs as well as myths around these issues.18,22,27,37,45,49,50 Menstruating women are perceived to be unclean, impure, and dirty by society in many cultures as well as by the menstruating women themselves.27,36,38 There are strict and absolute restrictions for participating in religious activities, for doing household chores, for touching family members or for sleeping in the same house, to cite a few examples.16,20 These studies provide a wealth of information, which can guide in designing interventions to improve menstrual hygiene management.

It is evident that countries have begun to include menstrual hygiene management in high-level policies, but this has not been accompanied by necessary protocols and directives to facilitate implementation.47 In Nepal, Menstrual Hygiene Management (MHM) challenges facing women and girls in Nepal have gained political attention: a policy on civil code of conduct against menstruation taboos and on dignified menstruation together with an MHM Master Plan (2018-2020) have been formulated. The government has already drafted a national policy on dignified menstruation and is in the process of endorsement by the Cabinet.51 The Supreme Court of Nepal issued a directive against the tradition in 2005 , but due to lack of legislation, chhaupadi was never criminalized. A new bill was passed by the parliament on 9 August 2017 to criminalize chhaupadi. The Criminal Code Bill passed by the Parliament on 9 August 2017 criminalizes an ancient practice that banishes women from the home during menstruation. The new law stipulates a three-month jail sentence or NPR 3000 fine, or both, for anyone forcing a woman to follow the custom. It also states that a woman during her menstruation or post-natal state should not be kept in chhaupadi or treated with any kind of similar discrimination or untouchable and inhuman behavior.52

Menstrual Hygiene Management must be viewed through a wide lens. For the MHM agenda to be effective and sustainable we cannot look at MHM as an add-on program; MHM must be mainstreamed and integrated into the wider efforts to promote girls' education. It implies that MHM is an integral component of the dialogue with policymakers, various stakeholders including communities.47 Policies and programs that address MHM must adopt a multifaceted approach by incorporating several interrelated issues, including water, sanitation and hygiene, gender sensitivity, cultural acceptability, financial barriers, as well as several sustainable methods. Several ministries and key players can work in tandem to ameliorate the situation of MHM around the globe, particularly in Low and Middle-Income Countries (LMICs). The Ministry of Education, Ministry of Health $(\mathrm{MOH})$ water authorities, school officials, non-governmental organizations (NGOs), community-based organizations, and parents have to work together in unison and common understanding. Schools should integrate MHM into their curriculums as a mandatory component. Teachers need to be trained and provided with educational materials on MHM. A greater extent of advocacy is required to address the taboo that exists around menstruation.

A lot can be done at the community level to achieve effective and sustainable MHM. The families of girls, community representatives, religious leaders, and other community members who are major influencers and can create a supportive and enabling environment for MHM can play a crucial role. Government ministries should collaborateat the community level 
for efficient mobilization of local resources to improve MHM. Non-state actors, including international nongovernment organizations (INGOs), non-government organizations (NGOs), and community-based organizations (CBOs), could also play a crucial role in generating awareness at the community level, providing technical and/or financial assistance for programs and promoting collaboration for sustainable MHM initiatives. Besides, the media could also be approached and utilized to develop a positive public opinion on MHM.

Despite the intensive literature search, we confined our search only to free accessed literature and limited paid accessed web pages. We confined our search only to literatures published from 2010 to March 2020. Hence, this paper may not cover all the published literature on knowledge of MHM. We looked at quantitative, qualitative andmixed method studies in this review. Studies conducted in South-East Asia, Middle East, African countries, LMICs were included in this review. For further assessment of the scientific literature on knowledge of MHM, we suggest more systematic reviews and meta-analyses studies.

\section{CONCLUSIONS}

The review concludes that the young girls are mostly not prepared for menstruation before menarche, and therefore menarche is a distressing experience of anxiety, fear, embarrassment, and pain; The girls are not provided with comprehensive information before menarche while growing up, and therefore the first experience of menstruation is without any preparation and usually comes as a shock.

The elders, teachers and parents believe that menstruation is a normal bodily function and there is nothing remarkable about it, and therefore girls do not need any special explanation or preparatory training for it. It is sad to note from the various studies that teachers and schools are doing very little to impart education on MHM to adolescents. A majority of the teachers themselves are least or unprepared to address this issue with the girls, even when it is part of the school curriculum.

The consequent misinformation, misconception and negative attitude and perception towards menstruation can be detrimental in the long run in the sexual and reproductive health journey of a young girl as well as in terms of social development and individual dignity and self-confidence.

These studies provide a wealth of information, which can guide in designing interventions to improve menstrual hygiene management. Extensive efforts to disseminate the accurate knowledge should be carried out to break the myths surrounding menstruation. Above all, the adolescent girl must be at the forefront and center stage at every level of involvement.

\section{REFERENCES}

1. Biran A, Curtis V, Gautam OP, Greenland K, Islam MS Schmidt W-P, et al. Menstrual Hygiene. In: Background paper on measuring WASH and food hygiene practices-definition of goals to be tackled post 2015 by the joint monitoring Programme. 1st ed. London: London School of Hygiene and Tropical Medicine; 2012. p. 81.

2. UNICEF. Guidance on menstrual health and hygiene. New York: UNICEF, 2019. https://www.unicef.org/wash/files/ UNICEF-Guidance-menstrual-health-hygiene-2019.pdf

3. Karki KB, Poudel PC, Rothchild J, PopeN, Bobin NC, Gurung Y, et al. Scoping Review And Preliminary Mapping: Men-strual Health and Hygiene Management in Nepal (pp. 1-96). $\quad$ https://maverickcollective.org/wpcontent/uploads/2017/06/ Scoping-Review-and-PreliminaryMapping-of-Menstrual-Health-in-Nepal.pdf

4. Poureslami M, Osati-Ashtiani F. Assessing knowledge, attitudes and behaviour of adolescent girls in sub-urban districts of Tehran about dysmenorrhea and menstrual hygiene, J Int Womens Stud. 2002;3(2):51-61.

5. Rembeck GI, M“oller M,Gunnarsson R. Attitudes and feelings towards menstruation and womanhood in girls at me- narche. Acta Paediatr. 2006;95(6):707-14

6. Bhore N, Kumbhar VR. Knowledge and practices regarding menarche and menstrual hygiene among the adolescent girls. Innov Pharma Pharmaco. 2014;2(3):359-64.

7. Kapoor A, Khari S. Knowledge, attitude and Socio-cultural beliefs of adolescent girls towards menstruation. J Nepal Pae-diatr Soc. 2016;36(1):42-9.

8. Mahon T, FernandesM. Menstrual hygiene in South Asia: A neglected issue for WASH (water, sanitation and hygiene) programmes . Gend Dev. 2010;18(1): 99-113.

9. Sommer M, Ackatia-Armah N, Connolly S, Smiles D. A com-parison of the menstruation and education experiences of girls in Tanzania, Ghana,Cambodia and Ethiopia. J Comp Int Edu. 2014;45(4):1-21.

10. Kuhlmann AS, Henry K, Wall LL. Menstrual Hygiene Management in Resource-Poor Countries. Obstet Gynecol Surv.2017;72(6):356-76.

11. Hennegan J, Shannon AK, Rubli J, Schwab KJ, MelendezTorres GJ. Women's and girls' experiences of menstruation in 
low- and middle-income countries: A systematic review and qualitative metasynthesis. PLOS MED. 2019;16:e1002803.

12. Sapkota D, Sharma D, Budhathoki SS, KhanalVK,Pokharel HP. Knowledge and practices regarding menstruation among school going adolescents of rural Nepal. J Kath Med Coll. 2013;2(3):122-8.

13. Neupane MS, Sharma K, Bista PA, Subedi S, Lamichhane S. Knowledge on menstruation and menstration hygiene practices among adolescents girls of selected Schools, Chitwan. J Chitwan Med Coll. 2020;10(31):69-73.

14. Ali ST, Rizvi NS. Menstrual knowledge and practices of female adolescents in urban Karachi, Pakistan. J Adolesc. 2010;33(4):531-41.

15. Santra S. Assessment of knowledge regarding menstruation and practice related to maintainance of menstrual hygiene among the women of reproductive age group in a slum of Kolkata, west Bengal, India. Int J Comm Med Public Health. 2017;4(3):708-12.

16. Shoor P. A study of knowledge, attitude, and practices of menstrual health among adolescent school girls in urban field practice area of medical college, tumkur. Indian J Health Sci Biomed Res. 2017;10(3):249-55.

17. Anee SU, Islam A, Wahid F, Ahmed K. Knowledge, attitude and practice on female Hygiene management among Madrasa girls in Dhaka, Bangladesh. Asian J Multidisciplinary Stud. 2020;3(1):110-6.

18. Tegegne TK, Sisay MM. Menstrual hygiene management and school absenteeism among female adolescent students in Northeast Ethiopia. BMC Public Health. 2014;14:1118.

19. Aluko OO, Modupe O, Ayomide O, Adebola O, Funmilola O, IriyiseOO. Knowledge and menstrual hygiene pratices among adolescents in senior secondary schools in ileife, South-West-ern Nigeria. J Water Sanitat Hyg Dev. 2014;4(2);248-256.

20. Morrison J, Basnet M, Bhatt A, Khimbanjar S, Chaulagai S, Shah N, et al. Girls' menstrual management in five districts of Nepal: Implications for Policy and Practice. Studies Soc Justice. 2018;12(2):251-72.

21. Rajbandari AK, Sagtani RA, GC K, Dhaubanjar R. Menstrual Hygiene Among adolescent school girls of BHaktapur, Nepal. Nepal Med Coll J. 2018;20(4):133-8.

22. Shah V, Nabwera MH, Sosseh F, Jallow Y, Comma E, Keita $\mathrm{O}$, et al. A rite of passage: a mixed methodology study about knowledge, perception and practices of menstrual hygiene management in rural Gambia. BMC Public Health. 2019;19(1):277.

23. Varghese M, Ravichandran L, Anandhan KA. Knowledge and practice of menstrual hygiene among adolescent girls. Ind J Youth Adol Health. 2015;2(3):36-43.

24. Mohamed ME, Mohamed GA, AL-Ajeal YL. Knowledge, beliefs and practices regarding menstruation among adolescent school girls in Seiyun City, Yemen. AAMJ. 2011;9(3):68-86.

25. Bano R, Sabhan F. Study of knowledge and practice of university females regarding reproductive Health and hygiene in Hail, Saudi Arabia. Int J Women's Health Repro Sci. 2015;3(1):31-9.

26. Gultie T, Hailu D, Workineh Y. Age of menarche and knowl-edge about menstrual hygiene management among adoles-cent school girls in Amhara province, Ethopia: Implication to health care workers and school teachers. PLoS One. 2014;9(9):1-9.

27. Yiadom BA, Aladago AD, Beweleyir J, Mohammad BH, Salifu FM, Azaarik M. Assessing the knowledge, attitude and practice of menstrual hygiene management among junior high schools adolescent females in the yendi municipality in the northern region of Ghana. Eur Sci J. 2018;14(36):468-87.

28. Kamboj N, Chandiok K. Knowledge and practices rearding menstrual hygiene among school going adolescent girls. J Curr Sci. 2019;20(1):1-8.

29. Yadav RN, Joshi S, Poudel R, Pandeya P. Knowledge, attitude, and practice on menstrual hygiene management among adolescents. J Nepal Health Res Counc. 2017;15(37):212-6.

30. Dahal A, Acharya PK. Effectiveness of information Education and Communication on Menstruation Hygiene Adolescent School Girls of Jumla District. J Nobel Med Coll. 2019;8(2):4-9.

31. Balat SM, Sahu KS, Patel M. Assessment of knowledge and practice of menstrual hygiene management among adolescent school girls in Ahmedabad City. Natl J Comm Med. 2019;10(1):30-34.

32. Ameade EPK, Garti HA. Relationship between female university students' knowledge on menstruation and their menstrual hygiene practices: A study in Tamale Ghana. Adv Prev Med. 2016;1056235.

33. Alam MU, Luby SP, Haider AK, Opel A, Shoab AK, Ghosh PK, et al. Menstrual hygiene management among Bangladeshi adolescent schoolgirls and risk factors affecting school absence: results from a cross-sectional survey. BMJ Open. 2017;7(7):1-10.

34. Upashe SP, Tekelab T, Mekonnen J. Assessment of practice of menstruation hygiene among high school girls in western Ethiopia. BMC Womens Health. 2015;15(84):1-10.

35. Raina D, Balodi G. Menstrual Hygiene: Knowledge, practice and Restrictions Among girls of Dehradun, uttarakhand, India. Glob J Interdiciplin Soc Sci. 2014;3(4):156-62.

36. Shrestha S, Kuikel S, Bhandari S. Menstrual hygiene among prison women in Kathmandu. Int $\mathrm{J}$ Health Sci Res. 2014;4(10):177-84.

37. Devis J, Macintyre A, Odagiri M, Suriatini W, Cordova A, Huggett $C$, et al. Menstrual hygiene management and school absenteeism among adolescent students in Indonesia: evidence from a cross-sectional school-based survey. Trop Med Int Health. 2018;23(12):1351-63.

38. Prajapati D, Shah J, Kedia G. Men-strual Hygiene: Knowledge and Practice among Adolescent Girls of Rural Kheda District. Natl J Community Med. 2015;6(3):349-53.

39. Pandit D, Bhattacharya P, Bhattacharya R. Menstrual Hygiene: Knowledge and Practice among Adolescent School Girls In rural areas of West Bengal. J Dent Med Sci. 2014;13(6):19-24

40. Rani SP. Knowledge and practices of menstrual hygiene among married adolescents and young Women in Chittoor District of Andra Pradesh, India. J Nursing and Health Sci. 2014;3(2):6-15.

41. Kaur S. Knowledge and attitude on menstrual hygiene among adolesent girls in Kapurthala District of Punjab. Int J Sci Res Rev. 2019;7(3):3374-9.

42. Dhingra R, Kumar A, Kour M. Knowledge and practices related to menstruation among Tribal (Gujjar) Adolescent Girls. Stud Ethno Med. 2009;3(1):43-8.

43. Thakre BS, Thakre SS, Reddy M, Rathi N, Pathak K, Ughade S. Menstrual Hygiene: Knowledge and Practice among Ado-lescent School Girls of Saoner, Nagpur District. J Clin Diag Res. 2011;5(5):1027-33.

44. Rumun JA, Msuega PA. Menstrual knowledge and practices among adolescent females in Makurdi Metropolis. Glob J In- 
terdisciplin Soc Sci. 2014;3(3):113-21

45. Thakur H, Aronsson A, Bansode S, Lundborg SC, Dalvie S, Faxelid E. Knowledge, practices and restrictions related to menstruation among young women from low socioeconomic community in Mumbai, India. Front Public Health. 20142:72.

46. Ramachandn K, Gilyaru S, Eregowda A, Yathiraja S. A study on knowledge and practices regarding menstrual hygiene among urban adolescent girls. Int $\mathbf{J}$ Contemp Pediatr. 2016;3(1):142-5

47. Girls in Control: Compiled Findings from Studies on Men strual Hygiene Management of Schoolgirls. 2014. [Internet] SNV Ethiopia. [Cited 2020 May 16]. Available at https://snv. org/cms/sites/default/files/explore/download/snv_girls_in_ control_baseline_report.pdf

48. Chinyama J, Chipungu J, Rudd C, Mwale M, Verstraete L, Sikamo C, et al. Menstrual hygiene management in ru-ral schools of Zambia:a descriptive study of knowledge, ex- periences and challenges faced by schoolgirls. BMC Public Health. 2019;19(1):16.

49. Lahme AM, Stern R, Cooper D. Factors impacting on menstrual hygiene and their implications for health promotion. Glob Health Promot. 2018;25(1):54-62.

50. Singh N, Sherpa AT, Pandey S, Pradhan A. Menstrual Hygiene: Practice and Challenges among Adolescent Girls of a Private School of Urban Nepal. Asian J Med Sci. 2019;10(4):39-43.

51. The Government of Nepal calls for dignified Menstrual Hygiene Management. [Internet]. WSSCC. Water Suppy\& Sanitation Collaborative Council. [Cited 2020 May 17]. Available at https://www.wsscc.org/2018/07/01/the-government-of-nepalcalls-for-dignified-menstruation-hygiene-management

52. Parliament in Nepal passes a bill to outlaw chhaupadi. [Inernet]. Save the Children. [Cited 2020 May 17]. Available at https:/campaigns.savethechildren.net/blogs/caitlinsmith/ parliament-nepal-passes-bill-outlaw-chhaupadi 